\title{
Article \\ Nanometers-thick Ferromagnetic Surface Produced by Laser Cutting of Diamond
}

\author{
Annette Setzer1 ${ }^{1}$, Pablo D. Esquinazi ${ }^{1 *}$ (D), Sergei Buga ${ }^{2,+}$, Milena T. Georgieva $^{1,+t}{ }^{+}$, Tilo Reinert ${ }^{3}$, Tom Venus ${ }^{4}$, Irina \\ Estrela-Lopis ${ }^{4}$, Andrei Ivashenko², Maria Bondarenko², Winfried Böhlmann' ${ }^{1}$ and Jan Meijer ${ }^{3}$
}

1 Division of Superconductivity and Magnetism, Felix-Bloch-Institute for Solid State Physics, University of Leipzig, 04103 Leipzig, Germany

2 Technological Institute for Superhard and Novel Carbon Materials, 7a Centralnaya street, Troitsk, Moscow 108840 Russia

3 Division of Applied Quantum Systems, Felix-Bloch-Institute for Solid State Physics, University of Leipzig, 04103 Leipzig, Germany

4 Institute of Medical Physics and Biophysics, University of Leipzig, D-04107 Leipzig, Germany

* Correspondence: esquin@physik.uni-leipzig.de

† Also at Moscow Institute of Physics and Technology, 9 Institutskiy per., Dolgoprudny, Moscow Region, 141701 Russia

$\ddagger$ On live from: Condensed Matter Physics and Microelectronics Division, Faculty of Physics, Sofia University St. Kl. Ohridski, James Bouchier Blvd 5, 1164 Sofia, Bulgaria

\begin{abstract}
In this work, we demonstrate that cutting diamond crystals with a laser (532 nm wavelength, $0.5 \mathrm{~mJ}$ energy, $200 \mathrm{~ns}$ pulse duration at $15 \mathrm{kHz}$ ) produces a $\lesssim 20 \mathrm{~nm}$ thick surface layer with magnetic order at room temperature. We have measured the magnetic moment with a SQUID magnetometer of six natural and six CVD diamond crystals of different size, nitrogen content and surface orientations. A robust ferromagnetic response at $300 \mathrm{~K}$ is observed only for crystals that were cut with the laser along the (100) surface orientation. The magnetic signals are much weaker for the (110) and negligible for the (111) orientations. We attribute the magnetic order to the disordered graphite layer produced by the laser at the diamond surface. The ferromagnetic signal vanished after chemical etching or after moderate temperature annealing. The obtained results indicate that laser treatment of diamond may pave the way to create ferromagnetic spots at its surface.
\end{abstract}

Keywords: Diamond; Magnetic order; Laser treatment

\section{Introduction}

Since the first studies on the magnetic order found in pure graphite-based samples were reported, see [1] and Refs. therein, the possibility of having magnetic order in other carbon-based compounds at room temperature and without doping with magnetic ions attracted the interest of the community. In case of pure diamond, Talapatra et al.[2] reported the existence of ferromagnetic hysteresis at room temperature in the magnetization of nanograins of diamond after nitrogen and carbon irradiation. This interesting result was attributed to structural modification or defects produced by the irradiation, a clear case of the phenomenon called defect-induced magnetism (DIM). In contrast to the ${ }^{12} \mathrm{C}$ implantation, a higher value of the magnetization at saturation was obtained after ${ }^{15} \mathrm{~N}$ implantation, which was attributed to the extra contribution of N-related centres in the diamond crystalline structure [2]. Superconducting (with a transition temperature of $T_{c} \sim 3 \mathrm{~K}$ ) and ferromagnetic (Curie temperature $T_{C}>400 \mathrm{~K}$ ) states were found in hydrogenated boron-doped nanodiamond films by Zhang et al.[3]. Narayan and Bhaumik reported ferromagnetic states after quenching carbon from an undercooled state using nanosecond laser pulses [4]. The observed magnetic state at room temperature depended on the energy and number of laser pulses and was 
attributed to a mixture of $\mathrm{sp}^{2}$-sp $\mathrm{s}^{3}$ bonds in the nanostructure of the diamond samples. Theoretical work studied the possibility of ferromagnetism in diamond taking into account disorder and certain doping [5]. Similarly to other carbon-based structures [6-13], H-atoms in diamond can have a finite magnetic moment, especially if they are located at the tetrahedral interstitial sites [5].

Taking into account the above mentioned work [2-5], we looked for the possibility to produce magnetic order at the surface of pure diamond samples via laser-induced graphitization. It has been known for almost 20 years, see [14] and Refs. therein, that the surface of pure diamond can be graphitized via laser pulses. The characteristics of the graphite structure at the surface of the diamond sample depends partially on the crystal orientation and length of the laser pulse $[14,15]$. Laser-induced graphitization has been also used to fabricate high quality-high conducting graphite wires embedded beneath the diamond surface [16]. In a recently published work, the effect of the cutting fluence (of a $532 \mathrm{~nm}$ wavelength laser with a pulse duration of $40 \mathrm{~ns}$ and a spot diameter of $40 \mu \mathrm{m}$ ) on CVD diamond surface was investigated with Raman and transmission electron microscopy (TEM) [17]. The authors found that the subsurface of the diamond samples shows a mixture of graphite and amorphous carbon and that the thickness of the graphite layer decreased with laser fluence.

In this study, we have used laser pulses of $532 \mathrm{~nm}$ wavelength, $0.5 \mathrm{~mJ} / \mathrm{cm}^{2}$ energy density and $200 \mathrm{~ns}$ pulse duration at $15 \mathrm{kHz}$ to produce a graphite layer at the surface of several diamond samples. Independently of the origin of the diamond sample, natural or CVD, we found that under the selected conditions, the laser pulses produced a magnetically ordered graphite film at $300 \mathrm{~K}$ of samples cut at the (100) oriented surface. Assuming a maximum thickness of $20 \mathrm{~nm}$ for the magnetic layer, the magnetization value at saturation varies from $\sim 10 \mathrm{emu} / \mathrm{g}$ to $20 \mathrm{emu} / \mathrm{g}$ at $300 \mathrm{~K}$, similar to the magnetization values obtained for defect-induced ferromagnetic graphite [1,11].

\section{Samples and Methods}

\subsection{Sample preparation: Laser cutting and after-cutting process of diamond crystals}

A single crystal of diamond is glued to the base surface of a mandrel, so that a large face is orientated perpendicular to the axis of the mandrel. Next, the mandrel (with the diamond crystal) is fixed in a device for the precise positioning in the laser-cut system. The system is equipped with a video camera that allows to adjust the face of the crystal to be cut along the axis of the laser beam to achieve the shortest laser cut length. The marking of the cut line on the selected face of the diamond single crystal is carried out on the computer monitor with the help of optical devices.

Before cutting, the laser beam was focused on the surface of the diamond at the level of the upper point of the cut. Then the laser beam was moved along the cut line, where the material was burned on the surface of the diamond with a width nearly the diameter of the laser beam focus. Thus, the working pass was performed at a certain depth in the crystal. After leaving the diamond sample, the laser beam was moved by a step (specified in the software) in the transverse direction and then moved in the opposite direction performing the next working pass. The heating of the diamond under the influence of laser radiation leads to graphitization, ablation and burn of carbon material $[14,15,18]$. By selecting the wavelength, power, and the duration of the laser radiation pulses, we can control the volume of material removal.

After performing several working steps, the laser beam reached half depth of the crystal to be cut. The shape of the cutting groove is wedge due to a conical shape of the focused laser beam. Thus, to reduce the amount of the ablated (burned) material, the mandrel with crystal is rotated $180^{\circ}$ and the cutting process continued from the back part of the crystal to cut its rest half of the thickness. To reduce the loss of diamond material during cutting, the cutting angle and the cutting width at the output are as small as possible. On the other hand, the laser beam must have a relatively large convergence angle to ensure high-quality focusing. With a significant reduction of the cutting angle, 
most of the laser power is reflected, which slows down the cutting process. Therefore, in order to optimize this process, we do a counter cut, which in turn helps to reduce the cutting depth and material losses. A SEM image of the obtained surface can be seen in Fig. 1(left).

After cutting, a polishing on the cut face was performed. The laser beam with the specified parameters, was focused right on the surface of the sample to burn part of the surface material produced by the cutting. A SEM image of the obtained surface can be seen in Fig. 1(right).

To remove the graphitic-like nanometers thick surface region we used two methods. For chemical etching the surface of the sample was treated with a mixture of $30 \mathrm{~mL}$ concentrated sulphuric acid $\left(\mathrm{H}_{2} \mathrm{SO}_{4}\right), 10 \mathrm{~mL}$ fuming salpetric acid $\left(\mathrm{HNO}_{3}\right)$ and $10 \mathrm{~mL}$ $70 \mathrm{vol} \%$ perchloric acid $\left(\mathrm{HClO}_{4}\right)$. This mixture was heated at $120 \mathrm{C}$ for $4 \mathrm{hs}$. under reflux. After cooling to room temperature, the acids were decanted and the diamond was intensively washed with distilled water and dried with nitrogen gas. A similar chemical procedure was used to remove any contaminants from the surface of the diamond samples. The other method we used is annealing the diamond sample in air at temperatures $T \leq 650 \mathrm{C}$ for a couple of hs.
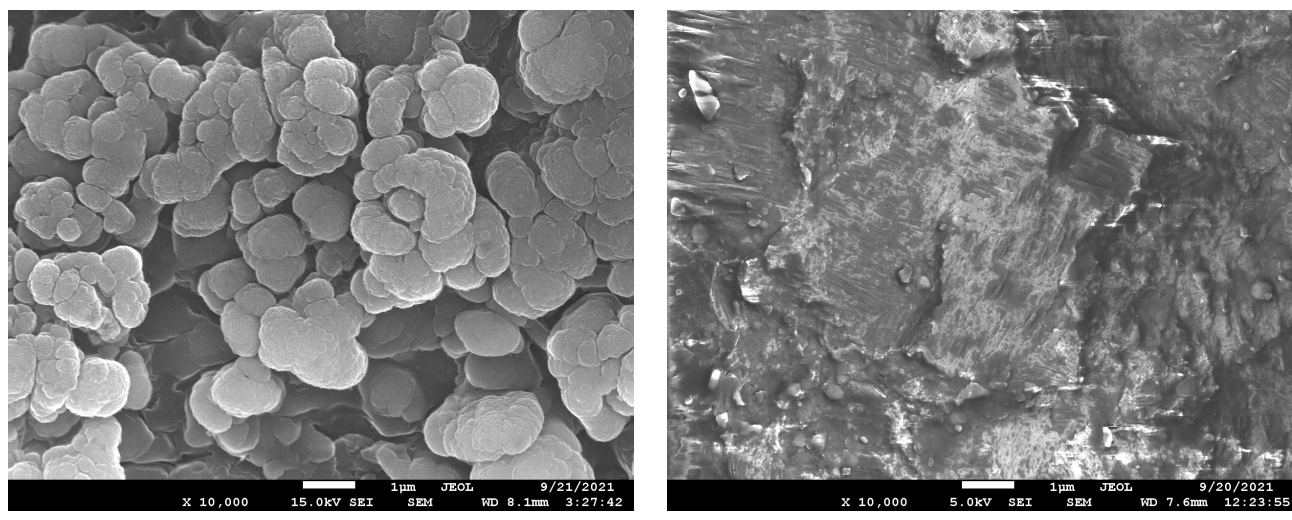

Figure 1. Scanning electron microscope images of the surface of a CVD diamond sample after laser cut (left image) and after laser polishing (right image)

\subsection{Samples characteristics}

\subsubsection{Natural diamond samples}

Table 1 shows several characteristics of the natural diamond samples like the nitrogen content and the concentration of $A, B$, and $C$ centres as well as the surface orientation of the laser cut surface. In Table 3 we include the concentration of the main magnetic impurities measured in the same samples.

Table 1. Size, orientation, total Nitrogen concentration $(\mathrm{N})$ and of $\mathrm{A}, \mathrm{B}$, and $\mathrm{C}$ centres of the natural diamond samples

\begin{tabular}{cccccc}
\hline Name & Orientation & $\begin{array}{c}\text { Mass } \\
\mathrm{mg}\end{array}$ & $\begin{array}{c}\text { Cut area } \\
\mathrm{mm}^{2}\end{array}$ & $\begin{array}{c}\mathbf{N} \\
\mathrm{ppm}\end{array}$ & $\begin{array}{c}\text { N-centres } \\
\mathrm{ppm}(\mathrm{A}, \mathrm{B}, \mathrm{C})\end{array}$ \\
\hline 354 & $(100)$ & 96.0 & $45 \pm 3$ & 3170 & $195,681,56$ \\
356 & $(100)$ & 106.0 & $15 \pm 3$ & 1400 & $522,78,46$ \\
379 & $(100)$ & 72.0 & $35 \pm 3$ & - & - \\
540 & $(100)$ & 96.0 & $25 \pm 3$ & 750 & $240,66,9$ \\
164 & $(111)$ & 38.6 & $20 \pm 3$ & 3900 & $605,656,81$ \\
384 & $(111)$ & 120.0 & $40 \pm 3$ & 2000 & $696,132,57$ \\
\hline
\end{tabular}




\subsubsection{CVD diamond samples}

As we will demonstrate below in this paper, the orientation of the laser cut surface plays a main role to produce the robust ferromagnetic nanometer thick surface region at room temperature. To support the results obtained from the natural diamond crystals, we have cut 6 CVD diamond samples at three orientations (100), (110) and (111), see Table 2. These samples have a total concentration of magnetic impurities below $2 \mathrm{ppm}$ and much lower $\mathrm{N}$ concentration than the one in the natural diamond samples. We measured the magnetic response of the CVD samples after the first cut and after polishing the cut surface with the laser beam. There are basically no large differences in the magnetic response between the two states, expect that after removing part of the cut surface by polishing, the ferromagnetic signal reduces in amplitude in the \#1 samples.

Table 2. Orientation, mass, and cut area of the CVD diamond samples. The states "cut" and "polish" refer to the first cut with the laser and the polishing done with the same laser afterwards, removing part of the graphitized surface. The total Nitrogen concentration of the CVD samples was $\lesssim 10$ ppm and $\lesssim 2$ ppm of magnetic impurities.

\begin{tabular}{ccccc}
\hline Name & Orientation & Laser treatment & $\begin{array}{c}\text { Mass } \\
\mathrm{mg}\end{array}$ & $\begin{array}{c}\text { Cut area } \\
\mathrm{mm}^{2}\end{array}$ \\
\hline 1a & $(100)$ & cut & 32.5 & $14.4 \pm 0.4$ \\
1b & $(100)$ & polish & 33.6 & $14.4 \pm 0.4$ \\
2a & $(110)$ & cut & 35.6 & $5.7 \pm 0.2$ \\
2b & $(110)$ & polish & 33.3 & $5.7 \pm 0.2$ \\
3a & $(111)$ & cut & 39.5 & $7 \pm 0.2$ \\
3b & $(111)$ & polish & 65.7 & $6.7 \pm 0.2$ \\
\hline
\end{tabular}

\subsection{Methods: PIXE, Raman and SQUID characterization}

The quantitative characterization of the main magnetic impurities ( $\mathrm{Fe}, \mathrm{Co}$ and $\mathrm{Ni}$ ) was done using Particle Induced X-ray Emission (PIXE) with protons. The parameters were, proton energy: $2.0 \mathrm{MeV}$, current: $2.5 \mathrm{nA}$, slit settings: Object/Aperture: $300 \mu \mathrm{m} / 300 \mu \mathrm{m}$, and a beam focus of $3 \mu \mathrm{m}$. Protons of $2 \mathrm{MeV}$ kinetic energy have a penetration range in diamond of about $25 \mu \mathrm{m}$. The X-ray production cross section decreases as the protons slow down inside the sample. Additionally, the contribution of X-rays to the detectable analytical signal decreases with the depth. However, this effect for X-rays from $\mathrm{Fe}, \mathrm{Co}, \mathrm{Ni}$ is less important due to the rather short range of $2 \mathrm{MeV}$ protons in carbon matrix.

Confocal Raman measurements were performed with the WiTec Alpha 300 System. A Laser wavelength of $532 \mathrm{~nm}$ (UHT S 300) was selected. A 50X objective (Zeiss, Germany) with a numerical aperture of 0.8 , a laser power at the sample surface of ca. $35 \mathrm{~mW}$ and a 1800 grating on the CCD detector with spectral resolution of ca. $0.8 \mathrm{~cm}^{-1}$ were used.

The measurements of the magnetic moment of the diamond samples have been done with a Superconducting Quantum Interferometer Device (SQUID) from Quantum Design. Magnetic field loops and temperature hysteresis were obtained after demagnetizing the samples at $380 \mathrm{~K}$. The time between two consecutive measurements at different fields or temperatures was $5 \mathrm{~min}$ or longer with similar results. No time dependence was detected within experimental resolution.

\section{Results}

\subsection{Magnetic impurities measurements}

From the characterization of the impurities with PIXE we conclude that the maximum magnetic impurity concentration was $2.6 \mathrm{ppm}$ of Fe in sample 164 . The sample 354 , which shows the largest magnetization at saturation, has a total concentration of magnetic impurities below $0.5 \mathrm{ppm}$, see Table 2 . 


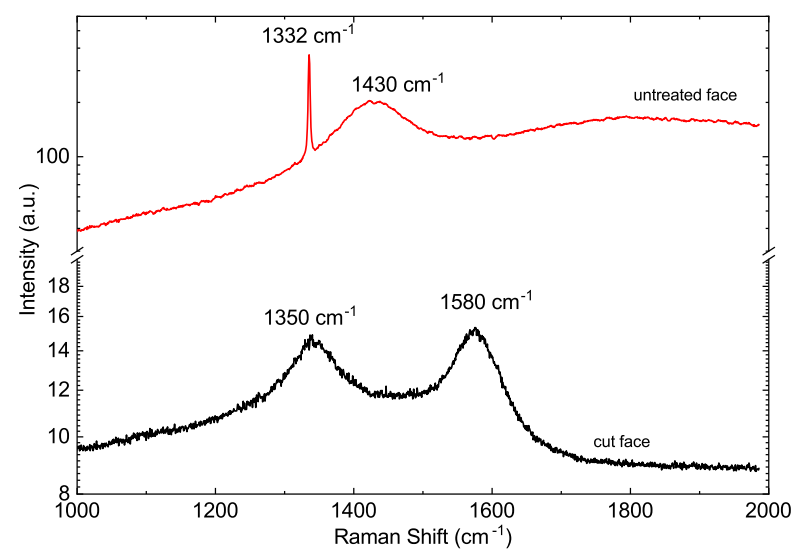

Figure 2. Raman Spectra obtained at room temperature for the untreated and laser cut faces of the CVD sample \#1b.

As example, let us estimate how large would be the contribution of $0.17 \mathrm{ppm}$ Fe in sample 354 to the magnetization at saturation. We assume that this small concentration of $\mathrm{Fe}$ or most likely magnetite, $\mathrm{Fe}_{3} \mathrm{O}_{4}$, behaves as a bulk ferromagnet with a saturation magnetization of about $100 \mathrm{emu} / \mathrm{g}$. The measured concentration of Fe in sample 354 would imply a ferromagnetic total mass of $76 \mathrm{ng}$, which in the unrealistic largest case could contribute with a magnetic moment of $4.8 \mu \mathrm{emu}$. This value is 4.2 times smaller than the magnetic moment at saturation measured at $300 \mathrm{~K}$ (see Section 3.3.1 below).

Table 3. Main magnetic impurities content (in $\mu \mathrm{g} / \mathrm{g}$ ) measured by Particle Induced X-ray Emission (PIXE) of the natural diamond samples. The selected areas for the measurements are included. MDL: minimum detectable limit.

\begin{tabular}{ccclcccc}
\hline Sample & Analyzed area & \multicolumn{3}{l}{ Concentration } & \multicolumn{3}{c}{ MDL } \\
& & Fe & Co & Ni & Fe & Co & Ni \\
\hline \multirow{2}{*}{354} & $(0.5 \mathrm{~mm})^{2}$ & 0.17 & 0.046 & 0.16 & 0.03 & 0.03 & 0.03 \\
& $(160 \mu \mathrm{m})^{2}$ & 0.08 & $<0.07$ & $<0.07$ & 0.05 & 0.04 & 0.05 \\
356 & spot & 1.99 & - & 0.12 & 0.07 & 0.04 & 0.05 \\
379 & $(0.5 \mathrm{~mm})^{2}$ & 1.85 & $<0.03$ & 0.22 & 0.04 & 0.03 & 0.04 \\
& $(240 \times 100) \mu \mathrm{m}^{2}$ & 0.72 & - & $<0.12$ & 0.1 & 0.1 & 0.1 \\
540 & $\mathrm{spot}$ & 0.57 & - & 0.11 & 0.06 & 0.04 & 0.04 \\
164 & $(0.5 \mathrm{~mm})^{2}$ & 2.6 & 0.038 & 0.098 & 0.04 & 0.03 & 0.03 \\
& $(75 \mu \mathrm{m})^{2}$ & 0.32 & - & $<0.17$ & 0.2 & 0.2 & 0.2 \\
384 & $(1 \mathrm{~mm})^{2}$ & 0.84 & 0.28 & 0.35 & 0.08 & 0.08 & 0.07 \\
& $(400 \mu \mathrm{m})^{2}$ & 0.8 & $<0.18$ & 0.17 & 0.2 & 0.2 & 0.2 \\
\hline
\end{tabular}

\subsection{Raman}

Raman measurements were done on both the untreated and laser cut surfaces of all samples. As example we show in Fig. 2 the results for the CVD sample \#1b. Whereas the untreated surfaces of the samples show a sharp absorption peak at $1332 \mathrm{~cm}^{-1}$ corresponding to pure diamond (first order Raman), the laser cut surfaces show disordered graphite-like peaks corresponding to the so-called G- $\left(1580 \mathrm{~cm}^{-1}\right)$ and D-band $\left(1350 \mathrm{~cm}^{1}\right)$, see Fig. 2. In case of the untreated CVD samples surfaces the peak at $1430 \mathrm{~cm}^{-1}$, known for CVD samples [19-21], is also clearly observed.

We observed some differences in the Raman patterns between the cut surfaces of the CVD "a" and "b" samples: whereas in the "b" samples only Raman peaks corresponding to disordered graphite were observed, the "a" cut surfaces showed also a weak signal 
coming from the diamond main Raman peak. Apparently, the polishing procedure transforms the rest of diamond-like regions left after the first laser treatment, leaving only disordered graphite regions. Within the experimentally observed broadening of the Raman peaks it is not possible to recognize systematic differences between the Raman spectra of the different cut surface orientations. Clearly, Raman characterization helps to identify the presence of graphite-like regions (after the laser treatment) but it does not provide clear hints for the presence of certain defects that can be correlated with the magnetic response.

\subsection{Magnetization measurements}

\subsubsection{Natural diamond samples}

In general, at $300 \mathrm{~K}$ and for the magnetic impurity concentration of our samples, natural diamond crystals do not show any sign of magnetic order within the resolution of our SQUID magnetometer $\left(\sim 5 \times 10^{-8} \mathrm{emu}\right.$ at an applied field of $\left.1 \mathrm{~T}\right)$. Nitrogen doping with concentrations measured in our samples, see Table 1, or lower does not trigger ferromagnetic order either. We note, however, that N-related C-centres are thought to be the origin for the clear hysteretic behavior in field and temperature observed below $50 \mathrm{~K}$ $[22,23]$. As we show below, the magnetic behavior of the untreated natural diamond crystals (or of the CVD samples) can be recovered after removing the graphitic-like film produced by the laser treatment.

Figure 3(a) shows the field hysteresis loops of sample 354 at $300 \mathrm{~K}$ before and after chemical etching and within $\pm 2 \mathrm{~T}$ field range. The same diamagnetic linear contribution was subtracted from both data sets. Before etching, the sample shows a clear ferromagnetic response with a magnetization (right y-axis) at saturation of $10 \mathrm{emu} / \mathrm{g}$, a value obtained assuming $20 \mathrm{~nm}$ thick magnetically ordered graphitized surface. We should note that this magnetization value is probably a minimum one because we assumed the largest possible magnetic thickness that the etching process can remove. In the inset of Fig. 3(a) we plot the difference between the FC and ZFC states at $0.01 \mathrm{~T}$ applied field. This difference follows a temperature dependence similar to that found in irradiated graphite [1,24].

To demonstrate the large difference in the ferromagnetic response between the cut sample before and after etching, the difference between the FC and ZFC states relative to the value in the ZFC state given by $100\left[m_{\mathrm{FC}}(T)-m_{\mathrm{ZFC}}(T)\right] /\left|m_{\mathrm{ZFC}}(T)\right|$ at different applied magnetic fields is shown in Fig. 3(b). In this figure we recognize that whereas this relative difference reaches $\sim 25 \%$ at low temperatures and at fields $\leq 0.02 \mathrm{~T}$ in the cut sample, it remains below $1 \%$ in the whole temperature range and applied fields after etching the sample.

Figure 4 shows the field hysteresis loops of four natural diamond samples with cut areas with orientation (100) (356 and 540, similar to sample 354, see Table 1) and with (111) orientation (164 and 384) at $300 \mathrm{~K}$. Taking into account the cut area and assuming the same ferromagnetic thickness in the four samples, we recognize that the ferromagnetic signals are clearly smaller for the (111) cut surface samples. This difference is not related to large differences in the assumed ferromagnetic mass because the cut surfaces are similar or their difference shifts the estimate value of magnetization in the opposite direction, see Table 1 . The ferromagnetic signal is not related to the total $\mathrm{N}$-concentration or to the concentration of three defect centres one finds in bulk $\mathrm{N}$-doped diamond (A, B, C), see Figs. 5(a) and (b). In this figure and due to the fact that these centres are distributed all over the sample, the shown magnetization values in Figs. 5(a) and (b) were obtained taking into account the whole sample masses.

\subsubsection{CVD diamond samples}

Figure 6(a) shows the field hysteresis loops measured at $300 \mathrm{~K}$ of all CVD samples, see Table 2, after subtracting the diamagnetic linear background. The results indicate a ferromagnetic behavior with a coercive fields of the order of 80 Oe for samples \#1a 

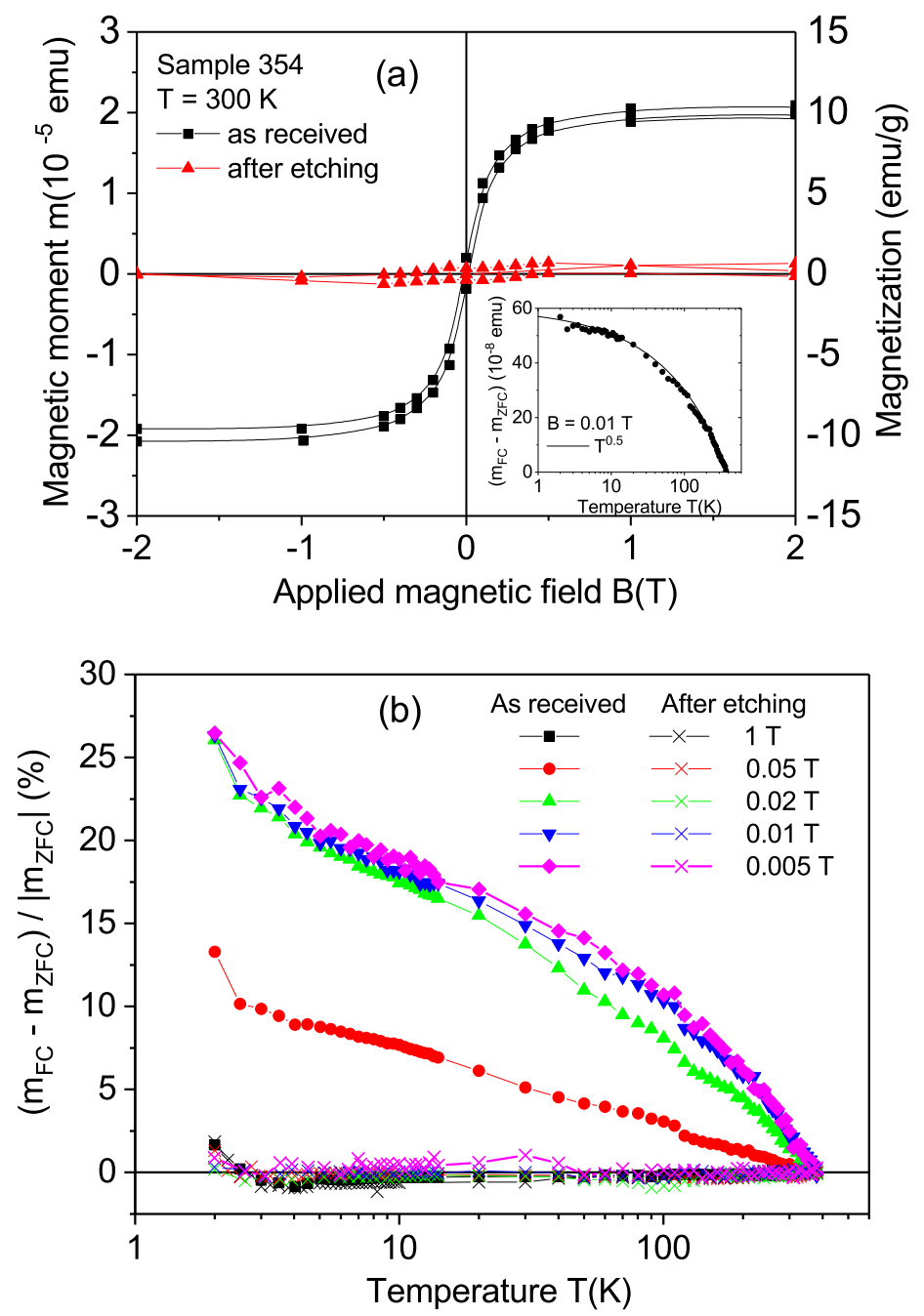

Figure 3. (a) Field hysteresis loops at $300 \mathrm{~K}$ of sample 354 in the as-received (after cutting plus polishing) state and after chemical etching removing $\lesssim 20 \mathrm{~nm}$ of the graphitized surface. The same linear diamagnetic background was subtracted from the measured curves. The inset shows the temperature dependence of the difference in the magnetic moment measured in the field cooled (FC) and zero-field cooled (ZFC) states measured at a fixed field of $0.01 \mathrm{~T}$. The continuous line follows the equation $m_{\mathrm{FC}}(T)-m_{\mathrm{ZFC}}(T)=10^{-8}\left(60-3 T^{0.5}\right)$ (emu). (b) Temperature dependence of the relative difference $100\left[m_{\mathrm{FC}}(T)-m_{\mathrm{ZFC}}(T)\right] /\left|m_{\mathrm{ZFC}}(T)\right|$ before and after etching. 


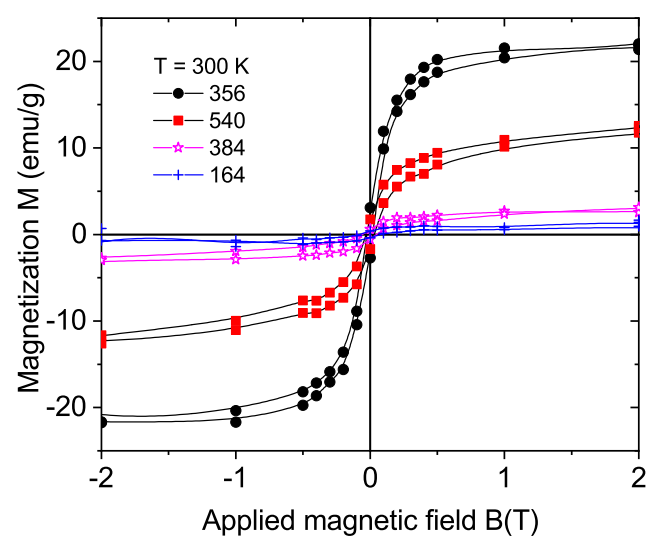

Figure 4. Field hysteresis loops at $300 \mathrm{~K}$ of two natural diamond cut on the (100) surface (356 and 540) and two others cut on the (111) surface (164 and 384). The magnetization values were calculated assuming a ferromagnetic mass given by a $20 \mathrm{~nm}$ thick region at the cut surfaces of the samples.

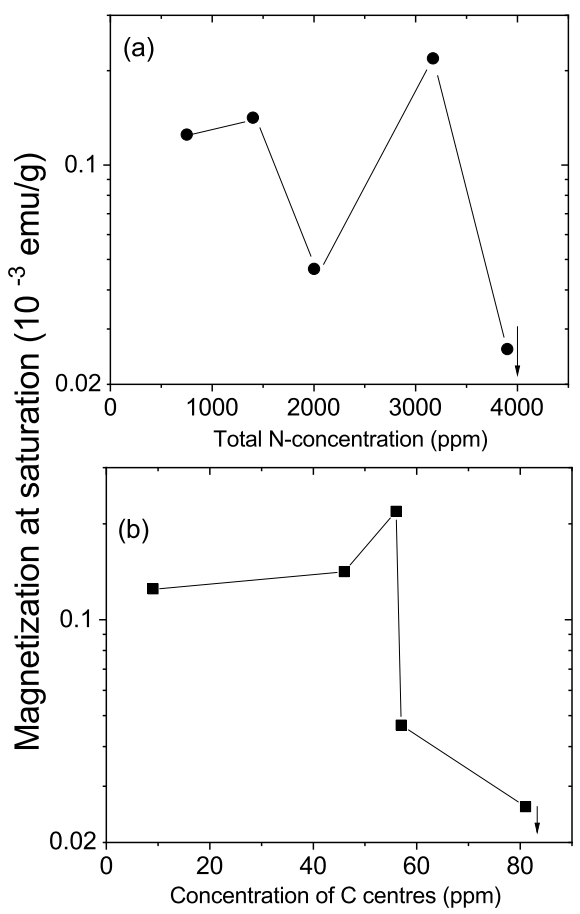

Figure 5. Magnetization at saturation at $300 \mathrm{~K}$ of the natural crystals vs. the total concentration of nitrogen (a) and of $\mathrm{C}$ centres. Because nitrogen is in all the sample volume, the magnetization has been calculated taking into account the total mass of each sample. The downarrow at the last point to the right means that the value of magnetization for that sample is below the limit of the $y$-axis. 

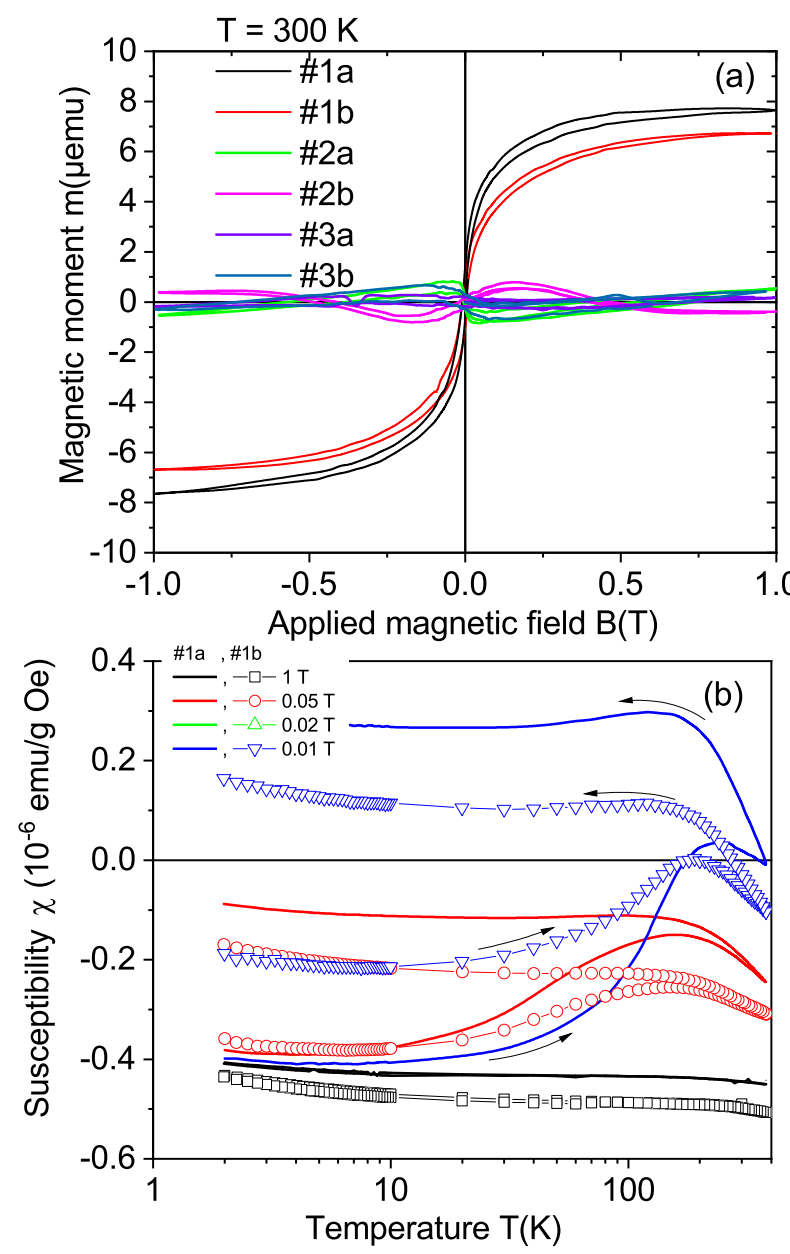

Figure 6. (a) Field hysteresis loops of the magnetic moment of all CVD samples at $300 \mathrm{~K}$. The diamagnetic background was subtracted from the measured data. (b) The susceptibility as a function of temperature at constant fields in the zero-field cooled (ZFC, warming) and field-cooled (FC, cooling) states of the two samples (\#1a: continuous lines, \#1b: symbols). No background was subtracted from the data. The mass used to calculate the susceptibility was the total sample mass, which is similar in both samples as well as the cut surface. 


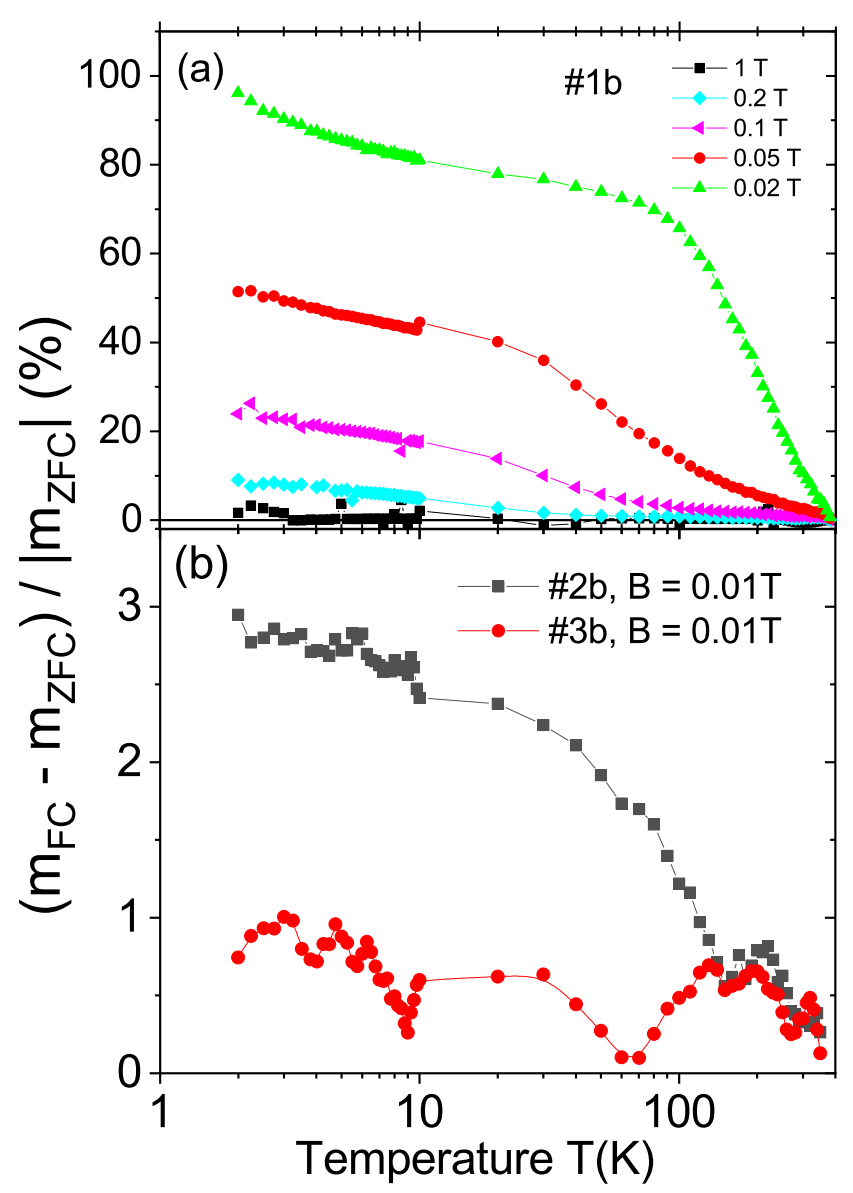

Figure 7. Temperature dependence of the relative difference $100\left[m_{\mathrm{FC}}(T)-m_{\mathrm{ZFC}}(T)\right] /\left|m_{\mathrm{ZFC}}(T)\right|$ for the CVD samples: (a) \#1b at five different applied fields and (b) \#2b and \#3b at a field of 0.01T. 
and $\# 1 b$, whereas the hysteresis loops, i.e. magnetic moment at saturation as well as the temperature and field hysteresis, of the other samples with the other orientations are much smaller. Taking into account the volume of the cut surface or the total mass, the obtained ferromagnetic magnetization of samples \#1a and \#1b is even larger that of the other CVD samples, supporting the orientation dependence of the laser cut surface observed in the natural diamond crystals. We note that the field loops obtained for some of the CVD samples are influenced by the hysteresis of the superconducting solenoid. This is a systematic error of the SQUID system that can influence the hysteresis loops after the background subtraction if the rest signals are below $1 \mu \mathrm{emu}$.

We observe that the saturation magnetic moment of sample \#1b is about $10 \%$ smaller than of sample \#1a. Because sample \#1b was obtained after laser polishing the cut surface, this difference in the magnetic moment for similar cut surfaces is expected. The ferromagnetic behavior (as well as the small difference between the samples) is clearly observed in the difference between zero-field cooled (ZFC) and field-cooled (FC states) as shown by the temperature dependence of the susceptibility, see Fig. 6(b). As expected, This difference as a function of temperature vanishes the higher the magnetic field in agreement with the field hysteresis shown in (a).

As observed in the natural diamond crystals, the samples cut with orientation other than the (100) show a much smaller or negligible ferromagnetic signal, see Fig.7. The relative difference between the FC and ZFC curves is nearly two orders of magnitude larger for the samples with (100) cut surfaces.

It is known that high temperature annealing in air removes any graphitic-like surface regions in diamond. Therefore, instead of using chemical etching to remove the graphitic surface of the CVD samples, as done in a natural diamond samples (see Fig. $3)$, we have annealed one of the CVD samples (\#1b) in air. The annealing procedure in air was $1 \mathrm{~h}$ at $550 \mathrm{C}, 1 \mathrm{~h}$ at $600 \mathrm{C}$ and $0.5 \mathrm{~h}$ at $650 \mathrm{C}$. Similarly to the result after chemical etching of a natural diamond sample, the ferromagnetic signal strongly decreased after annealing, see Fig. 8. All these results clearly indicate that the ferromagnetic signal comes from the disordered graphite surface region obtained after the laser cut and it is not related to magnetic impurities.

Before concluding, we would like to remark that the Curie temperature of the ferromagnetic order observed at the laser treated surfaces of diamond in the (100) direction is clearly larger than $380 \mathrm{~K}$. This temperature is the temperature at which we start with the FC measurement after the ZFC warming curve. This is the reason why the difference $m_{\mathrm{FC}}(T)-m_{\mathrm{ZFC}}(T)$ is always zero at the turning point temperature. A rough extrapolation of the observed temperature dependence of the magnetic moment to temperatures above $400 \mathrm{~K}$, see for example Figs. 4(b) and 6(b), indicates a Curie temperature between $500 \mathrm{~K}$ and $750 \mathrm{~K}$, similar to defect-induced ferromagnetic graphite, see [1] and Refs. therein.

\section{Conclusions}

The experimental results revealed that the laser cut surfaces of diamond with (100) orientation under the used conditions show robust ferromagnetism at room temperature. This magnetic order is clearly weaker or absent in the cases of the other two surface orientations. This fact indicates that during the laser-induced grow of the disordered graphite structure from diamond, certain lattice defects trigger the observed magnetic order. About the origin of ferromagnetism in graphite (without magnetic impurities) several experimental and theoretical studies were published in the last 20 years, for reviews see Refs. $[1,25,26]$. With a density of lattice defects or hydrogen between $5 \%$ and $10 \%$, graphite can be magnetically ordered with a strong spin polarized valence band, which affects the polarization of the barely occupied conduction band (graphite is a narrow-band-gap semiconductor $[27,28])$.

Further focused experimental characterization but also computer simulations [18] are necessary to find the lattice defect or defects (e.g., C-vacancies, $\mathrm{sp}^{2}-\mathrm{sp}^{3}$ or C-H 

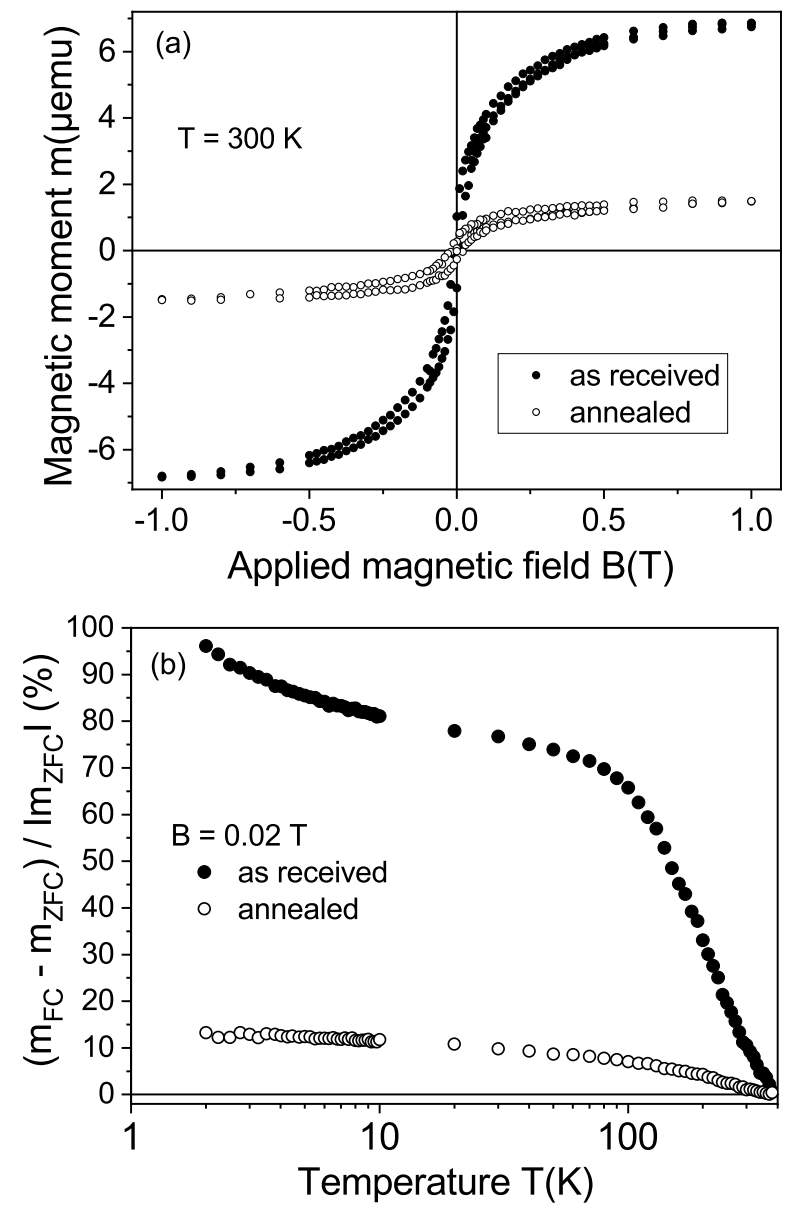

Figure 8. (a) Field hysteresis loops at $300 \mathrm{~K}$ obtained for the \#1b CVD sample in the as received state and after high temperature annealing. (b) Temperature dependence of the relative difference $100\left[m_{\mathrm{FC}}(T)-m_{\mathrm{ZFC}}(T)\right] /\left|m_{\mathrm{ZFC}}(T)\right|$ for the same sample before and after annealing at a field of $0.02 \mathrm{~T}$. 
complexes) responsible for the observed ferromagnetism. We have demonstrated that laser triggered a $\lesssim 20 \mathrm{~nm}$ thick ferromagnetic film at the (100) surface of diamond. Laser treatment can in principle be used to create localized magnetic spots of small area on a diamond surface. This phenomenon can be not only for memory devices of interest but also for other rather subtle applications, like using a localized magnetic spot near a nitrogen-carbon vacancy (NV-centre) to influence its magneto-optical response, especially to increase its field-sensitivity at certain applied field ranges.

Author Contributions: Conceptualization, P.D.E. and S.B.; methodology, A.S., P.D.E., S.B., M.T.G., T.V., I.E-L., T.R., A.I., M.B.; validation, A.S., P.D.E. and S.B.; analytics, T.R.; sample chemical cleaning, W.B.; resources, P.D.E.; data curation, A.S.; writing—original draft preparation, review and editing, P.D.E.; funding acquisition, J.M., S.B. and P.D.E. All authors have read and agreed to the published version of the manuscript.

Funding: This research was funded by the DFG under the grant DFG-ES 86/29-1 and DFG-ME 1564/11-1. The work in Russia was partially funded by RFBR and NSFC research project 20-5253051. The research stay of M.T.G. was supported by the DAAD under the Research Stays for University Academics and Scientists 2021 programme, Nr. 57552334.

Acknowledgments: One of the authors (PDE) gratefully acknowledges discussions with M. García (University of Kasel) and T. Lühmann (University of Leipzig). We thank N. Batova (Technological Institute) for the SEM measurements.

Conflicts of Interest: The authors declare no conflict of interest.

\section{Abbreviations}

The following abbreviations are used in this manuscript:

CVD Chemical Vapor Deposition

SQUID Superconducting Quantum Interference Device

PIXE Particle Induced X-ray Emission

\section{References}

1. Spemann, D.; Esquinazi, P., Chap. 3 in Basic Physics of Functionalized Graphite; Springer Series in Materials Science 244, P. Esquinazi (ed.), Springer International Publishing AG Switzerland, 2016; pp. 45-76. doi:https://doi.org/10.1007/978-3-31939355-1_3.

2. Talapatra, S.; Ganesan, P.G.; Kim, T.; Vajtai, R.; Huang, M.; Shina, M.; Ramanath, G.; Srivastava, D.; Deevi, S.C.; Ajayan, P.M. Irradiation-induced magnetism in carbon nanostructures. Phys. Rev. Lett. 2005, 95, 097201-1-4.

3. Zhang, G.; Samuely, T.; Xu, Z.; Jochum, J.K.; Volodin, A.; Zhou, S.; May, P.W.; Onufriienko, O.; Kačmarčík, J.; Steele, J.A.; Li, J.; Vanacken, J.; Vacík, J.; Szabó, P.; Yuan, H.; Roeffaers, M.B.J.; Cerbu, D.; Samuely, P.; Hofkens, J.; Moshchalkov, V.V. Superconducting Ferromagnetic Nanodiamond. ACS Nano 2017, 11, 5358-5366. doi:10.1021/acsnano.7b01688.

4. Narayan, J.; Bhaumik, A. Novel phase of carbon, ferromagnetism, and conversion into diamond. Journal of Applied Physics 2015, 118, 215303. doi:10.1063/1.4936595.

5. Kenmochi, K.; Sato, K.; Yanase, A.; Katayama-Yoshida, H. Materials Design of Ferromagnetic Diamond. Japanese Journal of Applied Physics 2004, 44, L51-L53. doi:10.1143/jjap.44.151.

6. Chan, J.A.; Montanari, B.; Gale, J.D.; Bennington, S.M.; Taylor, J.W.; Harrison, N.M. Magnetic properties of polymerized C 60 : The influence of defects and hydrogen. Phys. Rev. B 2004, 70, 041403(R)-1-4.

7. Kobayashi, Y.; Fukui, K.I.; Enoki, T.; Kusakabe, K. Edge state on hydrogen-terminated graphite edges investigated by scanning tunneling microscopy. Phys. Rev. B 2006, 73, 125415-1-8.

8. Ma, Y.; Lehtinen, P.O.; Foster, A.S.; Nieminen, R.M. Hydrogen-induced magnetism in carbon nanotubes. Phys. Rev. B 2005, 72, 085451-1-6.

9. Saito, T.; Ozeki, T.; Terashima, K. Hydrogen-induced magnetism in carbon nanotubes. Solid State Commun. 2005, 136, 546-549.

10. Lee, K.W.; Lee, Y.H.; Kim, I.M.; Lee, C.E. Magnetic Moment in Proton-Irradiated Graphite. Journal of the Korean Physical Society 2005, 47, 337-338.

11. Ohldag, H.; Esquinazi, P.; Arenholz, E.; Spemann, D.; Rothermel, M.; Setzer, A.; Butz, T. The role of hydrogen in roomtemperature ferromagnetism at graphite surfaces. New Journal of Physics 2010, 12, 123012. doi:http://stacks.iop.org/13672630/12/i=12/a=123012.

12. Makarova, T.L.; Shelankov, A.L.; Serenkov, I.T.; Sakharov, V.I.; Boukhvalov, D.W. Anisotropic magnetism of graphite irradiated with medium-energy hydrogen and helium ions. Phys. Rev. B 2011, 83, 085417. 
13. Friedman, A.L.; Chun, H.; Jung, Y.J.; Heiman, D.; Glaser, E.R.; Menon, L. Possible room-temperature ferromagnetism in hydrogenated carbon nanotubes. Phys. Rev. B 2010, 81, 115461. doi:10.1103/PhysRevB.81.115461.

14. Wang, C.Z.; Ho, K.M.; Shirk, M.D.; Molian, P.A. Laser-Induced Graphitization on a Diamond (111) Surface. Phys. Rev. Lett. 2000, 85, 4092-4095. doi:10.1103/PhysRevLett.85.4092.

15. Takesada, M.; Vanagas, E.; Tuzhilin, D.; Kudryashov, I.; Suruga, S.; Murakami, H.; Sarukura, N.; Matsuda, K.; Mononobe, S.; Saiki, T.; Yoshimoto, M.; ya Koshihara, S. Micro-Character Printing on a Diamond Plate by Femtosecond Infrared Optical Pulses. Japanese Journal of Applied Physics 2003, 42, 4613-4616. doi:10.1143/jjap.42.4613.

16. Sun, B.; Salter, P.S.; Booth, M.J. High conductivity micro-wires in diamond following arbitrary paths. Applied Physics Letters 2014, 105, 231105. doi:10.1063/1.4902998.

17. Mouhamadali, F.; Equis, S.; Saeidi, F.; Best, J.; Cantoni, M.; Hoffmann, P.; Wasmer, K. Nanosecond pulsed laser-processing of CVD diamond. Optics and Lasers in Engineering 2020, 126, 105917. doi:https:/ / doi.org/10.1016/j.optlaseng.2019.105917.

18. Jeschke, H.O.; Garcia, M.E.; Bennemann, K.H. Microscopic analysis of the laser-induced femtosecond graphitization of diamond. Phys. Rev. B 1999, 60, R3701-R3704. doi:10.1103/PhysRevB.60.R3701.

19. Stiegler, J.; von Kaenel, Y.; Cans, M.; Blank, E. Space filling by nucleation and growth in chemical vapor deposition of diamond. Journal of Materials Research 1996, 11, 716-726. doi:10.1557/JMR.1996.0086.

20. Badzian, A.; Badzian, T. Recent developments in hard materials. International Journal of Refractory Metals and Hard Materials 1997, 15, 3-12. Wear Resistant Materials for the South African Industry, doi:https:/ /doi.org/10.1016/S0263-4368(96)00047-9.

21. Zaitsev, A. Optical Properties of Diamond; Springer Berlin Heidelberg, 2001.

22. Barzola-Quiquia, J.; Stiller, M.; Esquinazi, P.; Molle, A.; Wunderlich, R.; Pezzagna, S.; Meijer, J.; Kossack, W.; Buga, S. Unconventional Magnetization below $25 \mathrm{~K}$ in Nitrogen-doped Diamond provides hints for the existence of Superconductivity and Superparamagnetism. Scientific Reports 2019, 9, 8743. doi:10.1038/s41598-019-45004-6.

23. Setzer, A.; Esquinazi, P.D.; Daikos, O.; Scherzer, T.; Pöppl, A.; Staacke, R.; Lühmann, T.; Pezzagna, S.; Knolle, W.; Buga, S.; Abel, B.; Meijer, J. Weak Electron Irradiation Suppresses the Anomalous Magnetization of N-Doped Diamond Crystals. physica status solidi (b) 2021, 258, 2100395. doi:https://doi.org/10.1002/pssb.202100395.

24. Esquinazi, P.; Barzola-Quiquia, J.; Spemann, D.; Rothermel, M.; Ohldag, H.; García, N.; Setzer, A.; Butz, T. Magnetic order in graphite: Experimental evidence, intrinsic and extrinsic difficulties. J. Magn. Magn. Mat. 2010, 322, $1156-1161$. doi:https://doi.org/10.1016/j.jmmm.2009.06.038.

25. Yazyev, O.V., Chap. 1 in Basic Physics of Functionalized Graphite; Springer Series in Materials Science 244, P. Esquinazi (ed.), Springer International Publishing AG Switzerland, 2016; pp. 1-24. doi:https://doi.org/10.1007/978-3-319-39355-1_1.

26. Ohldag, H., Chap. 2 in Basic Physics of Functionalized Graphite; Springer Series in Materials Science 244, P. Esquinazi (ed.), Springer International Publishing AG Switzerland, 2016; pp. 25-44. doi:https://doi.org/10.1007/978-3-319-39355-1_2.

27. García, N.; Esquinazi, P.; Barzola-Quiquia, J.; Dusari, S. Evidence for semiconducting behavior with a narrow band gap of Bernal graphite. New Journal of Physics 2012, 14, 053015. doi:10.1088/1367-2630/14/5/053015.

28. Ariskina, R.; Schnedler, M.; Esquinazi, P.D.; Champi, A.; Stiller, M.; Hergert, W.; Dunin-Borkowski, R.E.; Ebert, P.; Venus, T.; EstrelaLopis, I. Influence of surface band bending on a narrow band gap semiconductor: Tunneling atomic force studies of graphite with Bernal and rhombohedral stacking orders. Phys. Rev. Materials 2021, 5, 044601. doi:10.1103/PhysRevMaterials.5.044601. 